\title{
Rotating brush behaviour in duct cleaning
}

Rauno Holopainen ${ }^{\mathrm{a}}$, Eero-Matti Salonen ${ }^{\mathrm{b}}$

${ }^{a}$ Laboratory of Heating, Ventilating and Air Conditioning, Helsinki University of Technology HVAC-Laboratory, P.O.Box 4100,FIN-02015 HUT, Finland, e-mail: rauno.holopainen@hut.fi

${ }^{b}$ Laboratory of Structural Mechanics, Helsinki University of Technology, P.O.Box 2100, FIN-02015 HUT, Finland,e-mail: eero-matti.salonen@hut.fi

\begin{abstract}
A simple model of a rotating duct cleaning brush was presented using large deformation elastic theory. The results obtained by the model were compared with the results obtained by a laboratory test. Especially, the effect of air drag on the brush behaviour was considered.

The results show that with normal properties of a brush, air drag increases the torque needed and decreases somewhat the contact force between a bristle tip and the duct surface. However, air drag affects only slightly the deflections of the bristles at practical rotating speeds of the brush (300-1000 rpm) in a round duct. The modelled and experimental results for torque due to air drag were in good agreement in the case where the brush rotated without contact when a reduced air speed coefficient 0.5 was used in the simulation.
\end{abstract}

Keywords: Air drag; Bristle; Duct cleaning; Magnitude of normal force; Rotation speed of bristle; Torque 


\section{Introduction}

The ductwork and HVAC system components may become dirty from the construction process [1], or from dust and dirt accumulating in the system during its operation [2]. Dust and other contaminants may change the air delivery device into a source of contaminants. Beside health and hygienic problems, a dirty ventilation system may lead to increased energy consumption, increased cleaning costs and elevated risk of corrosion.

Mechanical brushing is commonly used as an efficient method for cleaning air ducts. The bristles of the brushes are selected according to the type of duct and to the composition of contaminants to be cleaned away. The rotating speeds of 300-1000 rpm are normally used during mechanical brushing [3]. The brushes are connected to a flexible whirling arm with variable length and the brushes are guided into the ducts with flexible rods. Ducts are normally brushed several times before an acceptable level of cleanliness is achieved.

The aim of the present study was to estimate the torque $T$ needed and to determine the value of the normal force $N$ acting at the bristle tip and the contact angle $\beta$ as a function of the rotation speed. In article [4], by the present authors, the governing equations describing the deformed shape of a rotating bristle are given. An unfortunate coding error was later found concerning the air drag integrals. The present article gives corrected results in this respect. In addition, an experimental arrangement is described and the results obtained are compared to those found by the simulation.

\section{Modelling}

The bristle of a rotating cleaning brush for air ducts was modelled using large deformation elastic theory [5]. Certain simplifying assumptions were made and these are described more thoroughly in the reference. A free-body diagram in Figure 1 describes the setting in some detail. The study of the 
bristle behaviour is performed in a $x y$-coordinate system with its origin at the rotation center and rotating with the attaching frame. The $x$-axis is along the undeformed straight bristle axis and the $y$-axis is 90 degrees in the clockwise direction according to the usual convention in the strength of the materials, e.g. the reference [6]. In this coordinate system the bristle is assumed to be in a static state and the motion is taken into account via centrifugal and air drag forces.

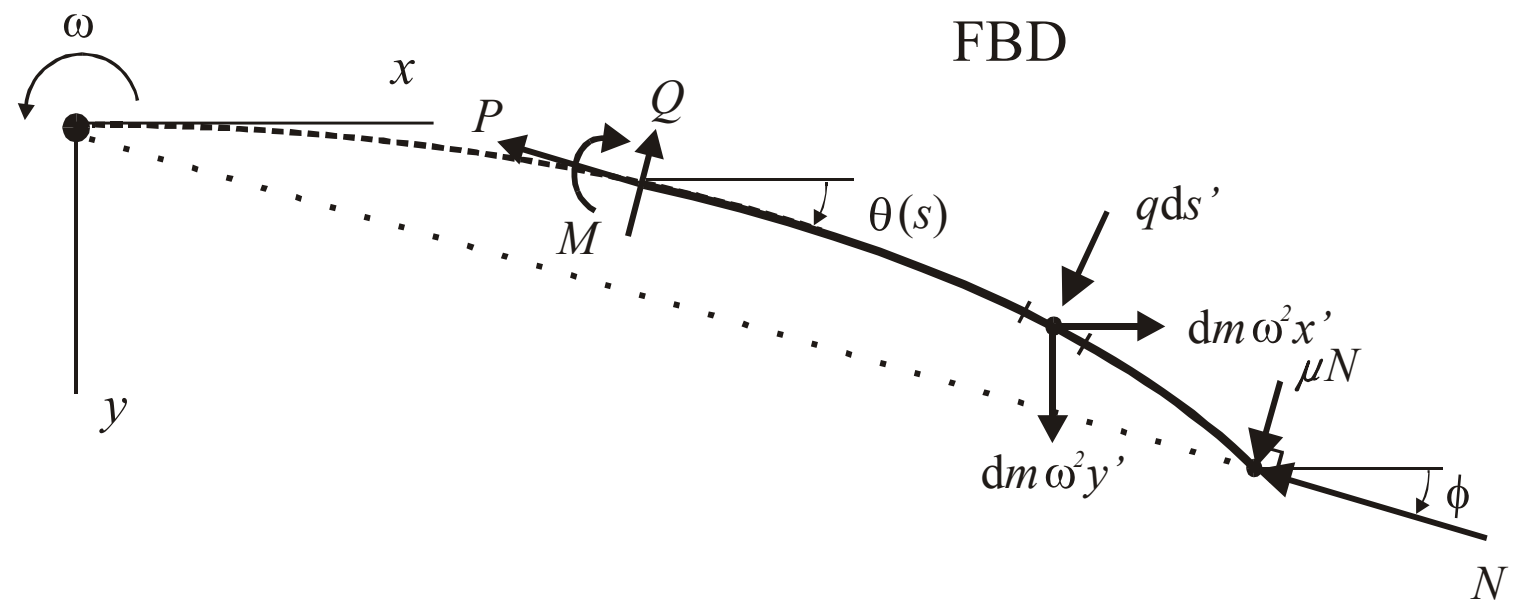

Fig. 1. A free-body diagram of a deformed bristle.

Referring to Figure 1 and article [4], we just repeat the equations derived there:

$N[\sin \theta(s) \cos \phi-\cos \theta(s) \sin \phi]+\mu N[\cos \theta(s) \cos \phi+\sin \theta(s) \sin \phi]-\rho A \omega^{2} \sin \theta(s) \int_{s}^{L} x^{\prime} \mathrm{d} s^{\prime}$

$+\rho A \omega^{2} \cos \theta(s) \int_{s}^{L} y^{\prime} \mathrm{d} s^{\prime}+\cos \theta(s) \int_{s}^{L} \cos \theta\left(s^{\prime}\right) q\left(s^{\prime}\right) \mathrm{d} s^{\prime}+\sin \theta(s) \int_{s}^{L} \sin \theta\left(s^{\prime}\right) q\left(s^{\prime}\right) \mathrm{d} s^{\prime}=-E I \frac{\mathrm{d}^{2} \theta}{\mathrm{d} s^{2}}$

$\theta(0)=0$,

$\frac{\mathrm{d} \theta}{\mathrm{d} s}(L)=0$,

$\sqrt{x^{2}(L)+y^{2}(L)}=R$.

The meaning of the notations is given in the Nomenclature section. The unknowns to be determined from equations (1) to (4) are the function $\theta=\theta(s)$ and the constant $N$. In addition, 
$x(s)=\int_{0}^{s} \cos \theta\left(s^{\prime}\right) \mathrm{d} s^{\prime}$,

$$
y(s)=\int_{0}^{s} \sin \theta\left(s^{\prime}\right) \mathrm{d} s^{\prime} .
$$

The evaluation of the terms due to air drag are described here in somewhat more detail than in reference [4]. The standard form of magnitude of the air drag per unit length for a cylinder with circular cross-section is [7]

$$
q_{\mathrm{N}}=\frac{1}{2} C_{\mathrm{D}} \rho_{\mathrm{a}} v^{2} d
$$

where $C_{\mathrm{D}}$ is the air drag coefficient, $\rho_{\mathrm{a}}$ the density of air, $v$ the speed of the cylinder with respect to air and $d$ the diameter of the cylinder; here of the bristle. The drag coefficient depends on the Reynolds number

$$
\operatorname{Re}=\frac{\rho_{\mathrm{a}} v d}{\mu_{\mathrm{a}}}
$$

where $\mu_{\mathrm{a}}$ is the viscosity of the air. The speed of a point of the bristle with respect to stagnant air is $\omega r\left(s^{\prime}\right)$, where

$$
r\left(s^{\prime}\right)=\sqrt{x^{2}\left(s^{\prime}\right)+y^{2}\left(s^{\prime}\right)}
$$

is the radial distance from the origin. The brush certainly sets the air around it to some motion which is difficult to estimate. In some effort to take this into account we evaluate the speed by $v=c \omega r\left(s^{\prime}\right)$

where $c$ is a dimensionless multiplier $(0<c \leq 1)$. If some experimental results are available, $c$ can be hopefully made use of. According to [8], when the flow is inclined to the axis of a cylinder, the air drag intensity perpendicular act to the axis can be evaluated from (we neglect the possible air drag component along the axis)

$q=q_{\mathrm{N}} \cos ^{2} \gamma$ 
where

$$
\gamma\left(s^{\prime}\right)=\theta\left(s^{\prime}\right)-\phi\left(s^{\prime}\right)
$$

is the angle between the bristle normal and bristle velocity vector. In more detail,

$$
\cos \gamma\left(s^{\prime}\right)=\cos \theta\left(s^{\prime}\right) \cos \phi\left(s^{\prime}\right)+\sin \theta\left(s^{\prime}\right) \sin \phi\left(s^{\prime}\right)
$$

and

$$
\begin{aligned}
& \cos \phi\left(s^{\prime}\right)=\frac{x\left(s^{\prime}\right)}{r\left(s^{\prime}\right)}, \\
& \sin \phi\left(s^{\prime}\right)=\frac{y\left(s^{\prime}\right)}{r\left(s^{\prime}\right)} .
\end{aligned}
$$

The air drag coefficient is evaluated by fitting a fourth degree polynomial function for a circular cylinder from measured data [7] as a function of the Reynolds number in logarithmic coordinates in the form $\log C_{\mathrm{D}}=f(\log \mathrm{Re})$. This gives [4]

$$
C_{\mathrm{D}}=10^{1.005-0.614 \log \operatorname{Re}+0.166(\log R e)^{2}-0.037(\log R e)^{3}+0.04819(\mathrm{Re})^{4}} .
$$

In the applications to follow, the Reynolds number stays under $10^{4}$, over which the expression (16) should not be used because of the non-smooth behaviour of $C_{\mathrm{D}}$.

\section{Solution Method}

A closed form solution of the problem described above is not possible to achieve. The unknown function $\theta=\theta(s)$ to be determined was approximated by a trial solution which is described in reference [5], as

$$
\theta(s) \approx \widetilde{\theta}(s)=\sum_{i=0}^{n} c_{i} s^{i}=c_{0}+c_{1} s+c_{2} s^{2}+c_{3} s^{3}+\ldots
$$

The point collocation method was used to determine the undetermined parameters $c_{0}, c_{1}, \ldots, c_{n}$. Here, the value $n=6$ was employed. The integrands were approximated first using Lagrangian interpolation with four evenly taken interpolation points. Five discrete equations following from (1) using evenly spaced collocation points are 


$$
\begin{aligned}
& N\left[\sin \widetilde{\theta}\left(s_{k}\right) \frac{\widetilde{x}(L)}{R}-\cos \widetilde{\theta}\left(s_{k}\right) \frac{\tilde{y}(L)}{R}\right]+\mu N\left[\cos \widetilde{\theta}\left(s_{k}\right) \frac{\widetilde{x}(L)}{R}+\sin \widetilde{\theta}\left(s_{k}\right) \frac{\tilde{y}(L)}{R}\right] \\
& -\rho A \omega^{2} \sin \widetilde{\theta}\left(s_{k}\right) \int_{s_{k}}^{L} \widetilde{x}\left(s^{\prime}\right) \mathrm{d} s^{\prime}+\rho A \omega^{2} \cos \widetilde{\theta}\left(s_{k}\right) \iint_{s_{k}}^{L} \tilde{y}\left(s^{\prime}\right) \mathrm{d} s^{\prime} \\
& +\cos \widetilde{\theta}\left(s_{k}\right) \int_{s_{k}}^{L} \cos \widetilde{\theta}\left(s^{\prime}\right) q\left(s^{\prime}\right) \mathrm{d} s^{\prime}+\sin \widetilde{\theta}\left(s_{k}\right) \int_{s_{k}}^{L} \sin \widetilde{\theta}\left(s^{\prime}\right) q\left(s^{\prime}\right) \mathrm{d} s^{\prime}=-E I \frac{\mathrm{d}^{2} \widetilde{\theta}}{\mathrm{d} s^{2}}\left(s_{k}\right), \quad k=1,2, \ldots, 5,
\end{aligned}
$$

where $s_{k}$ refers to the value of $s$ at collocation point $k$. The resulting equations are strongly nonlinear in the coefficients $c_{i}$. Condition (2) leads to

$$
\widetilde{\theta}(0)=c_{0}=0
$$

Condition (3) leads to

$$
\frac{\mathrm{d} \widetilde{\theta}}{\mathrm{d} s}(L)=c_{1}+2 c_{2} L+3 c_{3} L^{2}+\ldots=0 .
$$

These are both linear. Equation (4) gives

$$
\sqrt{\widetilde{x}^{2}(L)+\tilde{y}^{2}(L)}=R
$$

where $\tilde{x}$ and $\tilde{y}$ are integrated from $\tilde{\theta}(s)$ using (5) and (6) as explained in [5]. Concerning the integrals in equations (18), the terms due to centrifugal forces have been evaluated "directly" by substituting the involved terms $\tilde{x}\left(s^{\prime}\right)$ and $\tilde{y}\left(s^{\prime}\right)$ in them. However, the terms from air drag are so complicated that a direct approach is out of the question. Thus $q\left(s^{\prime}\right)$ is updated iteratively according to current achieved shape $\left(c_{0}, c_{1}, \ldots, c_{n}\right)$ of the bristle. The integrands $\cos \widetilde{\theta}\left(s^{\prime}\right) q\left(s^{\prime}\right)$ and $\sin \tilde{\theta}\left(s^{\prime}\right) q\left(s^{\prime}\right)$ are then represented by the Lagrangian interpolation functions and integrated analytically. This is an "indirect" approach, which could actually be used also for to the centrifugal forces. Usually about three iterations were needed to achieve practically converged results. The resulting non-linear algebraic system consisting of the eight equations (18), (19), (20) and (21) concerning the eight unknowns $c_{0}, c_{1}, c_{2}, c_{3}, c_{4}, c_{5}, c_{6}$ and $N$ is solved by a Mathcad [9] code. 
To start the solution, initial values for the unknowns must be given. The following were used: $c_{0}=0, c_{1}=0.5 / L, c_{2}=-0.25 / L^{2}, c_{3}=c_{4}=c_{5}=c_{6}=0$ and $N=0$.

\section{Laboratory test}

A rotating brush was tested in the Laboratory for Heating Ventilating and Air-Conditioning at Helsinki University of Technology. A round duct with a diameter $2 R=0.315 \mathrm{~m}$ was prepared of a metal sheet plate with width $0.2 \mathrm{~m}$. The diameter $2 L$ of the tested brush was $0.35 \mathrm{~m}$ and the bristles were nylon $\left(d=1 \times 10^{-3} \mathrm{~m}\right)$. The bristles were connected on a spiral frame made of metal wire. The number $n$ of the bristles was approximately 1000 . The brush was centralized at the center of the duct with the shaft of an electric motor $(0.55 \mathrm{~kW})$. In addition, certain tests were performed with just a pair of bristles. Figure 2 presents some details of the instrumentation of the laboratory test.

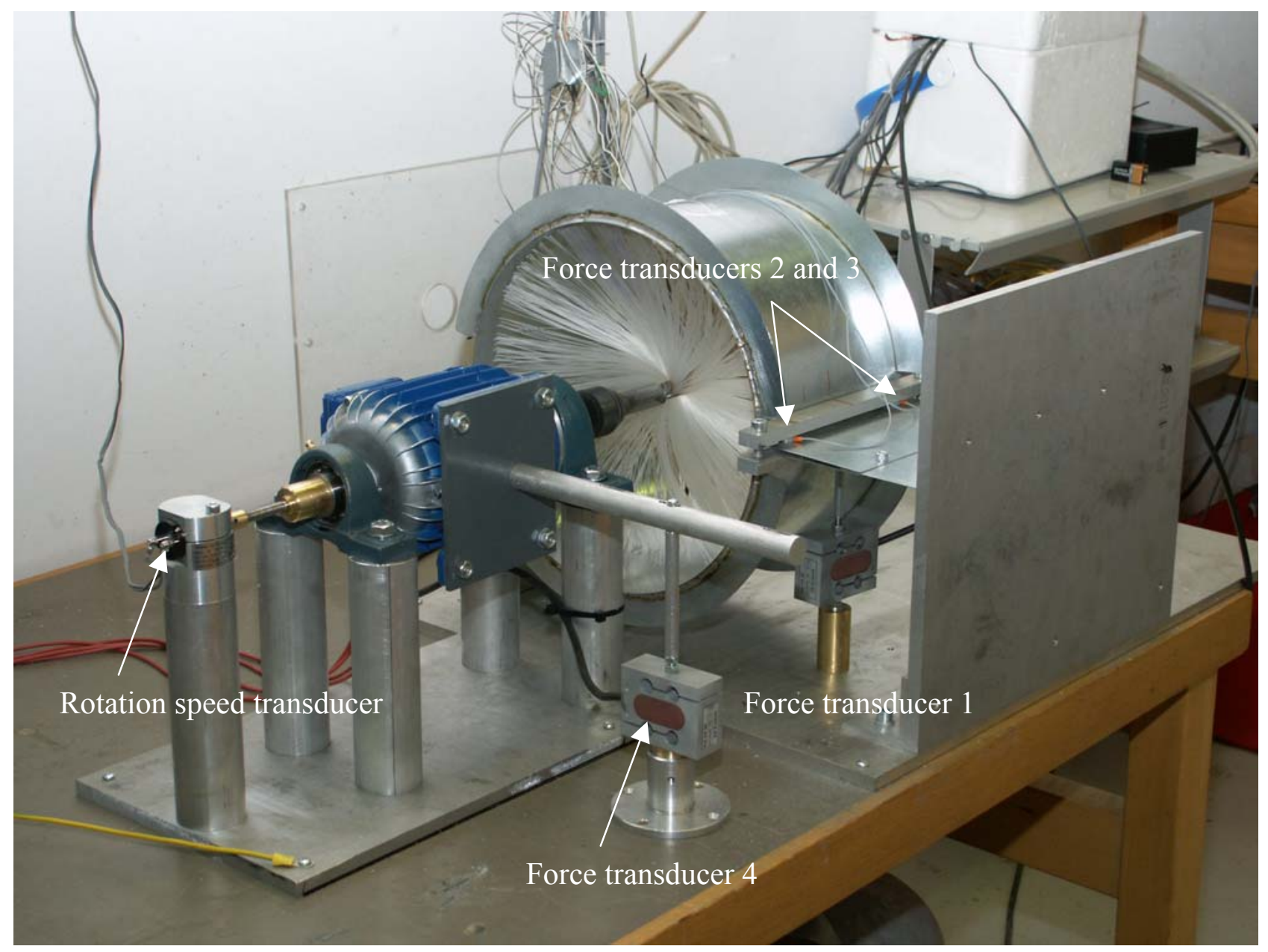

Fig. 2. Experimental arrangement in the laboratory. 
Measurements were obtained using force transducers 1, 2, 3 and 4. The range of transducers 1 and 4 were $0-118 \mathrm{~N}$ and of transducers 2 and 3 0-29 N. The transducers were located as indicated in Figures 2 and 3. The purposes of transducers 1 and 4 were to determine the torque (twisting moment) from the contact friction and the output torque from the electric motor, respectively. The output torque $T$ from the motor is considered to be balanced in general by the torque $T_{\mathrm{bf}}$ due to the bearing friction (some possible air drag acting on the shaft included) of the torque $T_{\text {ad }}$ due to the air drag from the brush (or from a bristle pair) and of the torque $T_{\mu}$ due to the contact friction of the brush (or of a bristle pair) with the duct surface; thus in general $T=T_{\mathrm{bf}}+T_{\mathrm{ad}}+T_{\mu}$. The motor output torque is obtained by

$T=P b$,

where $P$ (positive when transducer 4 is in tension) is the force measured by transducer 4 and $b=0.227 \mathrm{~m}$ is the horizontal distance between the centroid of the motor shaft and transducer 4 (Figure 3). Torque $T_{\mathrm{bf}}$ for a given rotation speed is obtained by rotating the motor alone, so then $T_{\mathrm{bf}}=T$. When the brush rotates with contact, the total output torque $T=T_{\mathrm{bf}}+T_{\mathrm{ad}}+T_{\mu}$. Assuming $T_{\mathrm{bf}}$ known with a given rotation speed, we can then determine $T_{\mathrm{ad}}+T_{\mu}=T-T_{\mathrm{bf}}$ from the experiment. Further, $T_{\mu}$ can be obtained using transducer 1 as described below from equation (27), so finally we can determine $T_{\text {ad }}$ from

$T_{\mathrm{ad}}=\left(T-T_{\mathrm{bf}}\right)_{4}-\left(T_{\mu}\right)_{1}$,

where the meaning of the notations is obvious.

To have some estimate of the effect of air drag without the inclusion of contact forces, torque due to air drag was measured separately rotating the brush in "air" meaning that the duct was removed. In addition, the brush was rotated without contact in a duct with a diameter of $0.365 \mathrm{~m}$. Here 
$T=T_{\mathrm{bf}}+T_{\mathrm{ad}}$ and we can determine $T_{\mathrm{ad}}$ assuming again $T_{\mathrm{bf}}$ known for a given rotation speed.

However, this torque

$T_{\mathrm{ad}}=T-T_{\mathrm{bf}}$

is naturally not the same as the torque $T_{\text {ad }}$ obtained by (23) with a brush in contact with the duct.

Further, experiments were performed using single pairs of bristles without trying to employ the transducers as the forces involved would be too small for proper measurements. Instead, deflections of the bristles were recorded by taking photos by a digital camera. Preliminary laboratory tests with a rotating brush were performed in the Laboratory for Mechanics of Materials at Helsinki University of Technology [10]. The experience obtained with these tests was useful for setting up the present laboratory arrangement.

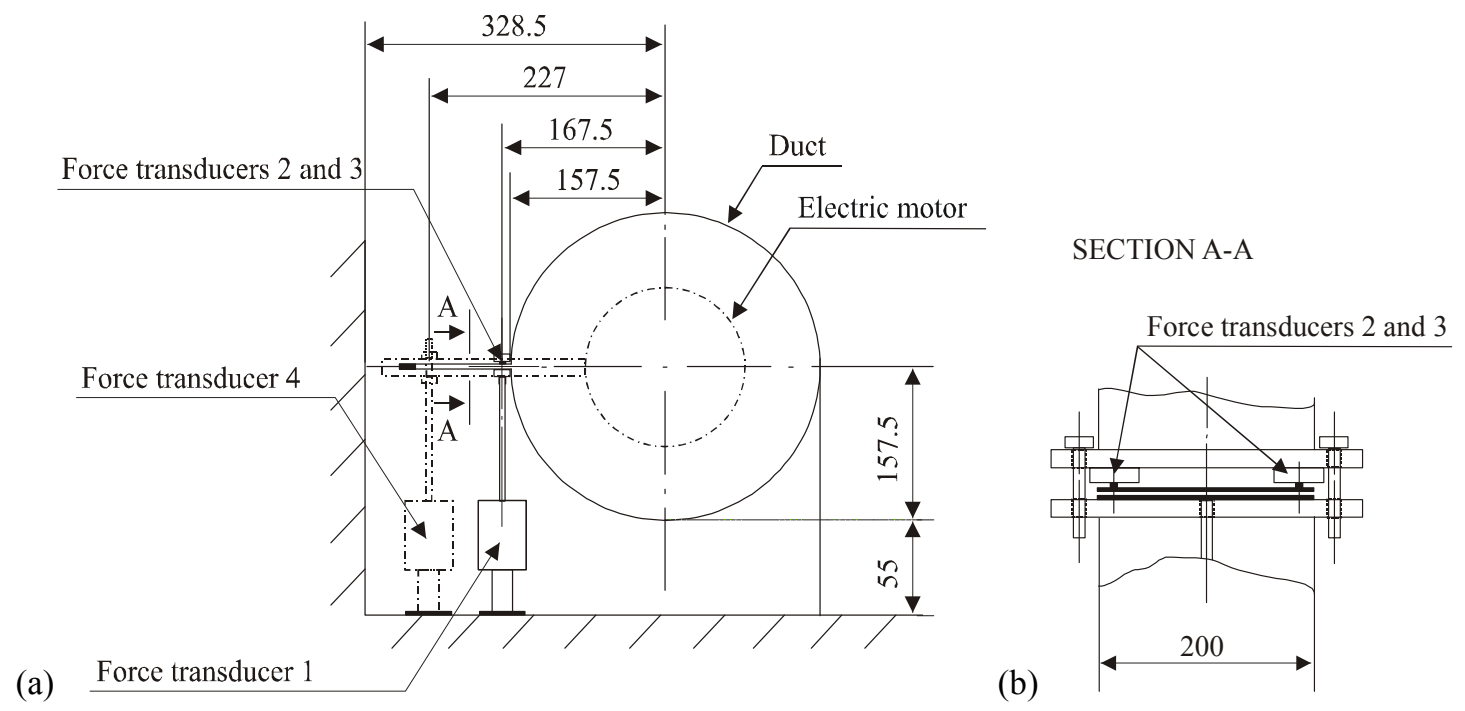

Fig. 3. (a) Instrumentation of the laboratory test. (b) Detail of the transducers installed in the seam of the duct frame at the left hand side. Length units are given in $\mathrm{mm}$.

The purpose of transducer 2 and 3 was to measure the "opening force" in the seam of the duct frame. Two free-body diagrams of the duct frame used in the analysis of the measurement results with transducers 1, 2, and 3 are shown in Figure 4 [5]. The frame is looked similarly as in Figure 3 
from the opposite direction of the motor and the rotation direction of the motor and the brush shown in the diagrams is considered here to be in the counterclockwise direction.
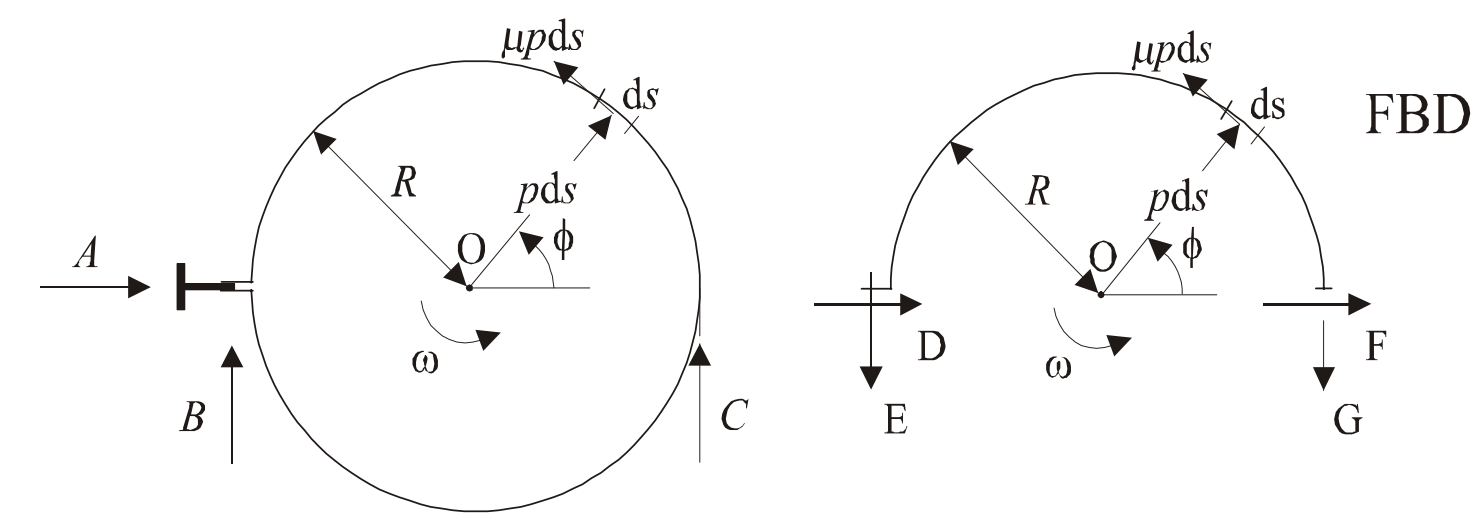

(a)

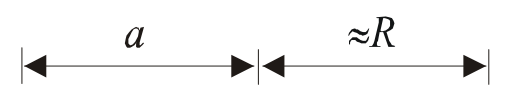

(b)

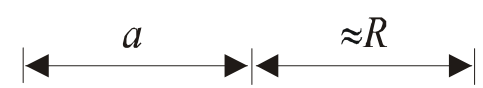

Fig. 4. (a) Free body-diagram of the duct frame. (b) Free body-diagram of the upper part of the duct frame $(a=0.1675 \mathrm{~m}$ and $R=0.1575 \mathrm{~m})$.

It is assumed that the plates supporting the duct frame are very flexible compared to the rigidity of the duct frame and thus the possible shearing forces and bending moments in the plates are neglected in the free-body-diagrams. Force $B$ (positive when transducer 1 is in compression) and opening force $E$ (positive when transducers 2 and 3 are in tension) in the diagrams are measured in the test by transducer 1 and as the sum of the values of 2 and 3 together, respectively. The effect of the bristle normal forces $N$ is represented by a distributed line loading per unit length $p=n N /(2 \pi R)$, where $n$ is the number of bristles and $R$ the inner radius of the duct. The possible effect of air drag on the duct is neglected. The final expressions obtained for the bristle contact normal force and the coefficient of friction from the equilibrium equations using the free-body diagrams are [5]

$$
\begin{aligned}
& N=\left(\frac{a}{R}+1\right)\left(\frac{B}{2}+E\right) \frac{\pi}{n}, \\
& \mu=\frac{1}{\left(\frac{1}{2}+\frac{E}{B}\right) \pi} .
\end{aligned}
$$


Further, the torque from the brush contact friction is

$T_{\mu}=B(a+R)$.

The calculations performed above are based on assuming the rotation direction of the brush to be in the counterclockwise direction. If the rotation direction is in the clockwise direction, we obtain the expressions (we denote the quantities now with a dash) [5]

$$
\begin{aligned}
& N^{\prime}=\left(\frac{a}{R}+1\right)\left(\frac{B^{\prime}}{2}+E^{\prime}\right) \frac{\pi}{n}, \\
& \mu^{\prime}=-\frac{1}{\left(\frac{1}{2}+\frac{E^{\prime}}{B^{\prime}}\right) \pi}, \\
& T_{\mu}^{\prime}=B^{\prime}(a+R) .
\end{aligned}
$$

The maximum rotation speeds that could be reached with the brush in contact with the duct were about $1200 \mathrm{rpm}$. With a bristle pair the corresponding value was about $3000 \mathrm{rpm}$.

\section{Results}

\subsection{Simulation details}

In the calculations described below the bristle cross section was taken to be circular and length $L=0.175 \mathrm{~m}, \quad$ diameter $\quad d=1 \times 10^{-3} \mathrm{~m}, \quad$ cross-sectional moment of inertia $I=\pi d^{4} / 64=4.909 \times 10^{-14} \mathrm{~m}^{4}$, density $\rho_{\mathrm{b}}=1140 \mathrm{~kg} / \mathrm{m}^{3}$, friction coefficient $\mu=0.7$, kinematic viscosity of air $v_{\mathrm{a}}=1.528 \times 10^{-5} \mathrm{~m}^{2} / \mathrm{s}$, bristle length duct radius ratio, $L / R=1.111(R=0.1575 \mathrm{~m})$ and Young's modulus $E=2.8 \mathrm{GPa}$. The values for $\mu$ and $E$ were selected roughly according to the results of the present laboratory tests (Figure 14) and [11], respectively.

\subsection{Rotating bristle calculations}

\subsubsection{Effect of air drag on deflection of rotating single bristle without contact}


Figure 5 shows calculated deflection of bristles with the rotation speed of $0 \mathrm{rpm}, 500 \mathrm{rpm}$, $1000 \mathrm{rpm}$ and $2000 \mathrm{rpm}$ without contact with air drag $(c=1$, see equation (10)). Note that Mathcad uses coordinates so that the $y$-direction is upwards (the rotation direction of the bristle is clockwise). The simulation model was obtained from the original model with contact simply by discarding equation (21). Correspondingly, the unknown $N$ disappears from the formulation. Without air drag the calculated shape of the bristle is naturally irrespective of the rotation speed always straight.

(a)

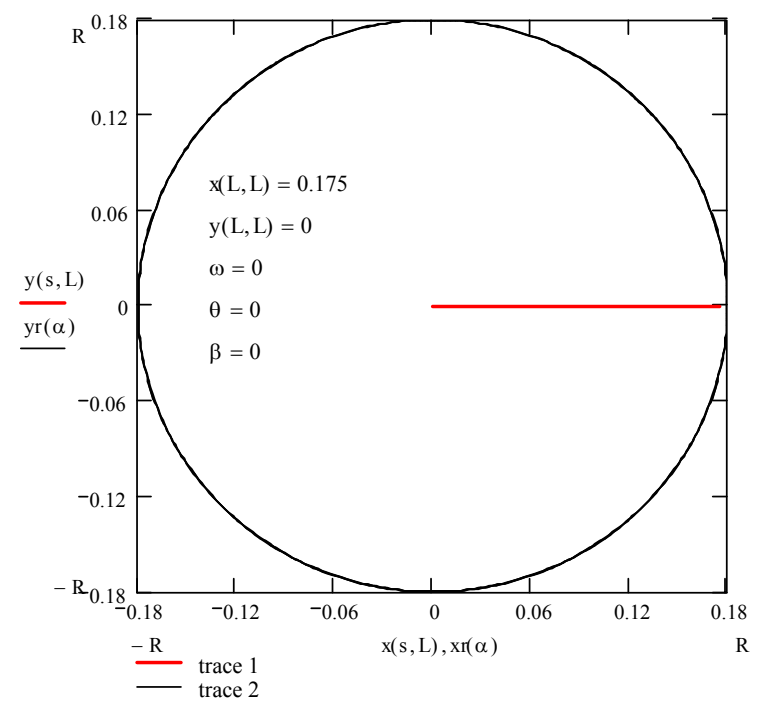

(b)

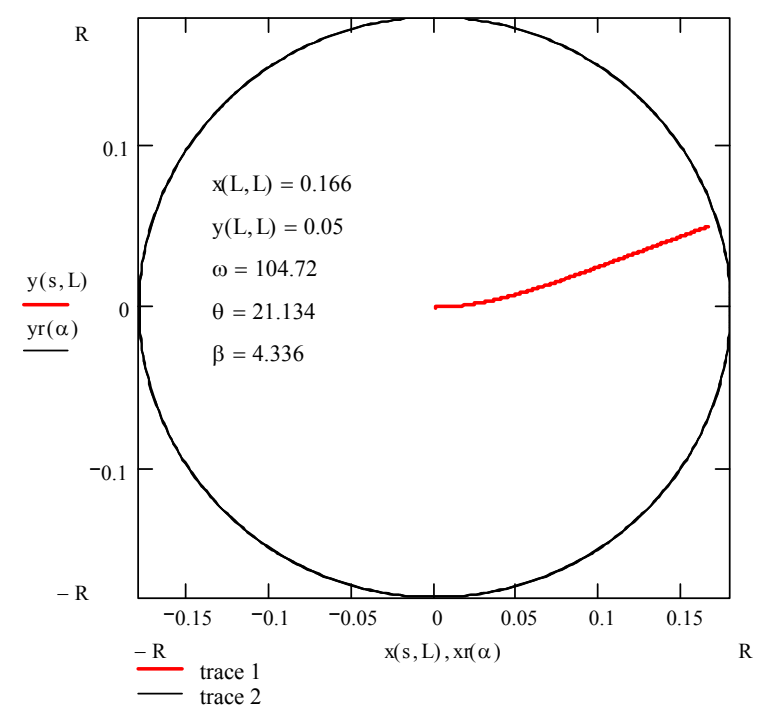

(c)

(d)
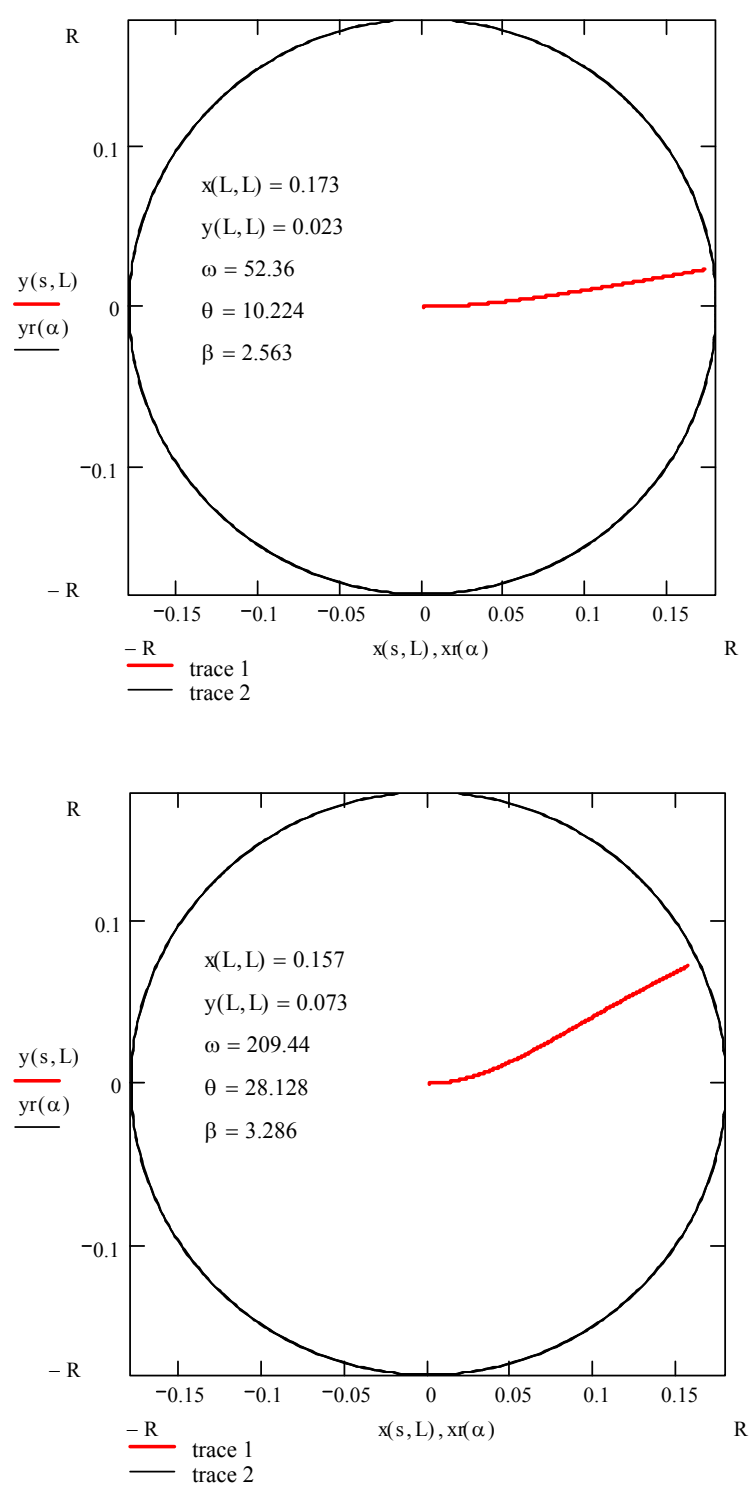

Fig. 5. Deflected form of the bristle (a) bristle rotation speed $n=0 \mathrm{rpm}$, (b) $n=500 \mathrm{rpm}$, (c) $n=1000 \mathrm{rpm}$ and (d) $n=2000 \mathrm{rpm}$ in air. 
As to be expected, air drag deforms the bristle most near the origin. Also, with higher rotation speeds the centrifugal forces seem to straighten somewhat the bristle near the free end.

The bending moment in a bristle at the origin evaluated from the deformed shape is $-E I \mathrm{~d} \theta(0) / \mathrm{d} s$. Taking into account the sign conventions used, the torque from a single bristle evaluated from this is thus (superscript symbol $*$ is used here and in the following for the torque from one bristle to discern it from torque from a brush)

$T_{\mathrm{b}}^{*}=E I \frac{\mathrm{d} \widetilde{\theta}}{\mathrm{d} s}(0)$.

Another way to evaluate the torque due to air drag from a single bristle is to use the integral

$T_{\mathrm{ad}}^{*}=\int_{0}^{L} q\left(s^{\prime}\right) r\left(s^{\prime}\right) \cos \gamma\left(s^{\prime}\right) \mathrm{d} s^{\prime}$

The integrands were approximated applying Lagrangian interpolation at four interpolation points $s_{1}=0, s_{2}=L / 3, s_{3}=2 L / 3$ and $s_{4}=L$. Table 1 presents the intensity $q\left(s^{\prime}\right)$, angle $\theta(L)$, contact angle $\beta$, torque $T_{\mathrm{b}}^{*}$ and torque $T_{\mathrm{ad}}^{*}$.

Table 1

Intensity $q\left(s^{\prime}\right)$, angle $\theta$, contact angle $\beta$, torque $T_{\mathrm{b}}^{*}$ and torque $T_{\mathrm{b}}^{*}$ due to air drag with $c=1$ when a bristle rotates without contact as a function of rotation speed $n$

\begin{tabular}{lrrrr}
\hline Parameter & \multicolumn{1}{l}{$n$} & \multicolumn{2}{l}{$n$} \\
& $500 \mathrm{rpm}$ & $1000 \mathrm{rpm}$ & $1500 \mathrm{rpm}$ & $2000 \mathrm{rpm}$ \\
\hline$q_{1}(\mathrm{~N} / \mathrm{m})$ & 0 & 0 & 0 & 0 \\
$q_{2}(\mathrm{~N} / \mathrm{m})$ & 0.008 & 0.028 & 0.057 & 0.097 \\
$q_{3}(\mathrm{~N} / \mathrm{m})$ & 0.028 & 0.100 & 0.215 & 0.371 \\
$q_{4}(\mathrm{~N} / \mathrm{m})$ & 0.059 & 0.218 & 0.473 & 0.831 \\
$\theta\left(^{\circ}\right)$ & $3.0^{\mathrm{a}}$ & $6.1^{\mathrm{a}}$ & $7.4^{\mathrm{a}}$ & $7.4^{\mathrm{a}}$ \\
& 10.2 & 21.1 & 26.4 & 28.1 \\
$\beta\left(^{\circ}\right)$ & $0.7^{\mathrm{a}}$ & $1.2^{\mathrm{a}}$ & $1.2^{\mathrm{a}}$ & $0.8^{\mathrm{a}}$ \\
& 2.6 & 4.3 & 4.2 & 3.3 \\
$T_{\mathrm{b}}^{*}(\mathrm{Nm})$ & 0.00046 & 0.00153 & 0.00292 & 0.00450 \\
$T_{\mathrm{ad}}^{*}(\mathrm{Nm})$ & $0.00014^{\mathrm{a}}$ & $0.00047^{\mathrm{a}}$ & $0.00101^{\mathrm{a}}$ & $0.00173^{\mathrm{a}}$ \\
& 0.00047 & 0.00170 & 0.00366 & 0.00636 \\
\hline $\mathrm{a}$
\end{tabular}


Intensity $q\left(s^{\prime}\right)$, and torque $T_{\text {ad }}^{*}$ increase roughly quadratically as a function of the rotation speed in the interval 0-2000 rpm. Without discretization errors the torques $T_{\mathrm{b}}^{*}$ and $T_{\mathrm{ad}}^{*}$ should be here in theory equal: $T_{\mathrm{b}}^{*}=T_{\mathrm{ad}}^{*}$. The values of $T_{\mathrm{ad}}^{*}$ are probably much more trustworthy as they are integrated quantities; $T_{\mathrm{b}}^{*}$ gives a local result, which is sensitive to small changes in the value of the parameter $c_{1}$. The results for $T$ (with $c=1$ ) are approximately of the same order of magnitude (difference $\leq 10 \%$ ) when the rotation speed is under $1000 \mathrm{rpm}$.

\subsection{Effect of air drag on deflection of rotating single bristle in duct}

Figure 6 shows simulated deflection of bristles without $(c=0)$ and with air drag $(c=1)$ in duct. The rotation speed of the bristle is $500 \mathrm{rpm}$ and $2000 \mathrm{rpm}$. 


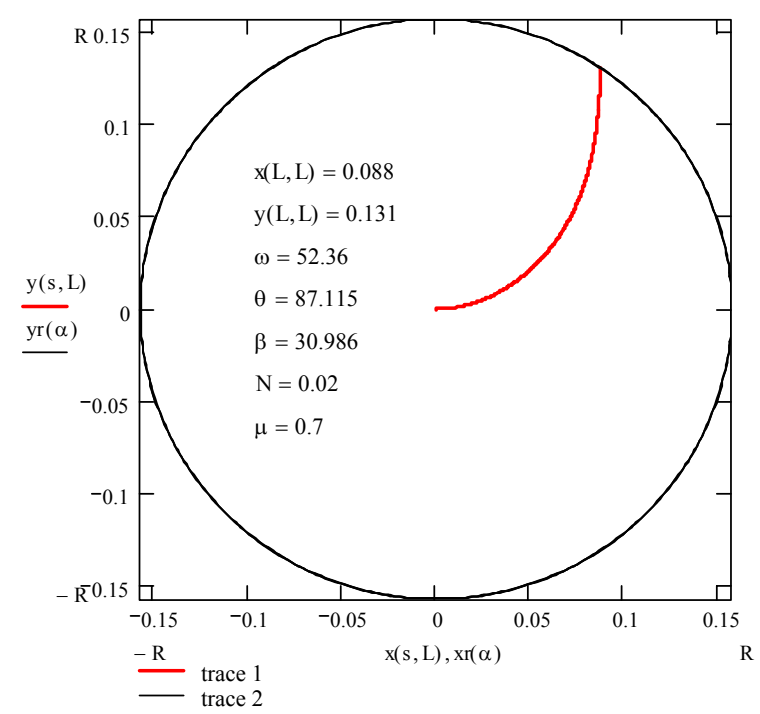

(a)

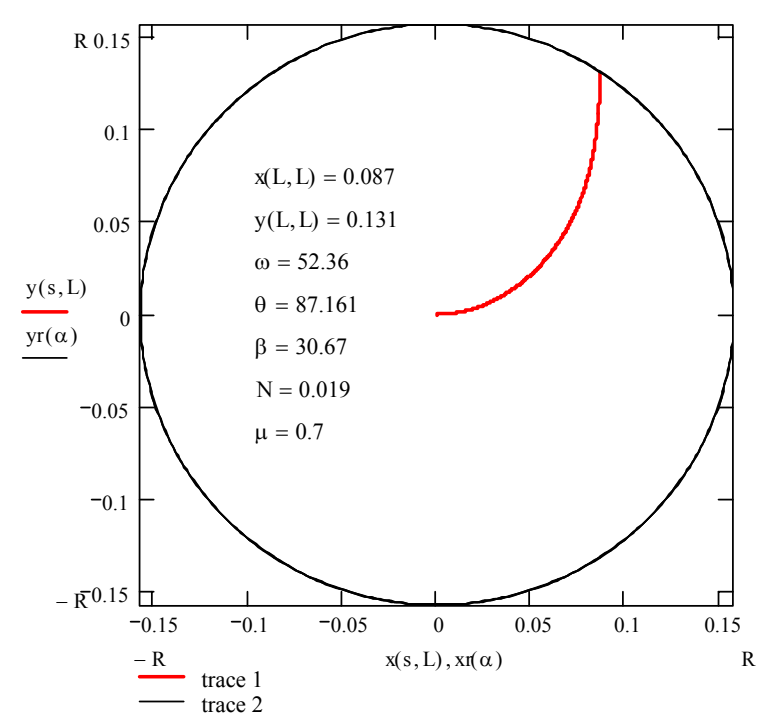

(b)
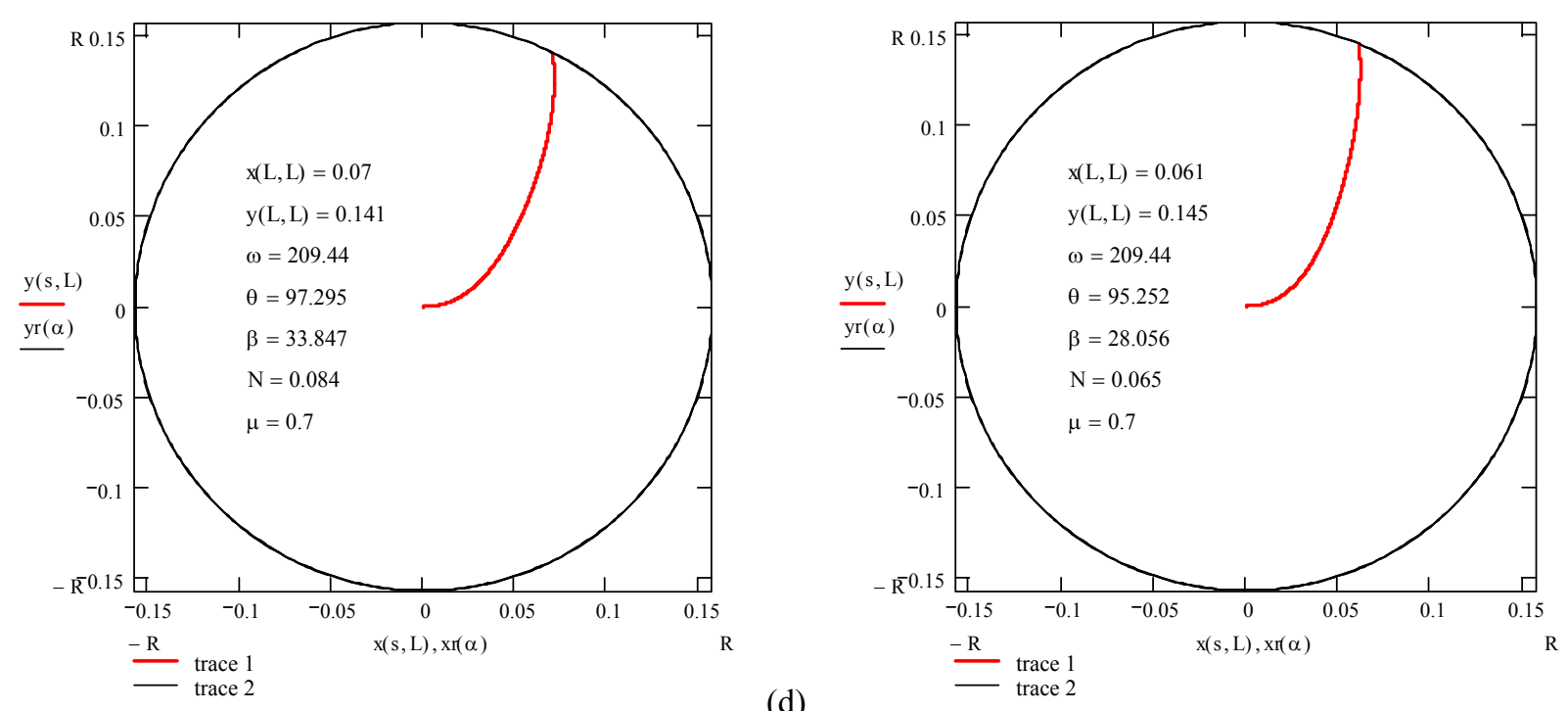

(d)

Fig. 6. Deflected form of the bristle. (a) The bristle rotates at the speed $n=500 \mathrm{rpm}$ without air drag and (b) with air drag. (c) The bristle rotates at the speed $n=2000 \mathrm{rpm}$ without air drag and (d) with air drag.

The deflection of bristle in the duct (with parameters described in Chapter 5.1) does not change significantly due to air drag at the rotation speeds of 0-2000 rpm. However, air drag decreases somewhat both the magnitude of the normal force $N$ and the contact angle $\beta$.

The quantities of $q\left(s^{\prime}\right), N, \theta(L), \beta, T_{\mathrm{b}}^{*}, T_{\mu}^{*}$ due to friction and torque $T_{\mathrm{ad}}^{*}$ due to air drag are presented in Table 2. The torque from the friction forces for a single bristle is calculated from the expression 
$T_{\mu}^{*}=\mu N R$

Table 2

Intensity $q\left(s^{\prime}\right)$, normal force $N$, angle $\theta$, contact angle $\beta$, torque $T_{\mathrm{b}}^{*}$, torque $T_{\mu}^{*}$ and torque $T_{\text {ad }}^{*}$ with $c=1$ when a bristle rotates with contact in duct as a function of rotation speed $n$

\begin{tabular}{|c|c|c|c|c|c|}
\hline Parameter & $\begin{array}{l}n \\
0 \mathrm{rpm}\end{array}$ & $\begin{array}{l}n \\
500 \mathrm{rpm}\end{array}$ & $\begin{array}{l}n \\
1000 \mathrm{rpm}\end{array}$ & $\begin{array}{l}n \\
1500 \mathrm{rpm}\end{array}$ & $\begin{array}{l}n \\
2000 \mathrm{rpm}\end{array}$ \\
\hline$q_{1}(\mathrm{~N} / \mathrm{m})$ & 0 & 0 & 0 & 0 & 0 \\
\hline$q_{2}(\mathrm{~N} / \mathrm{m})$ & 0 & 0.007 & 0.023 & 0.046 & 0.076 \\
\hline$q_{3}(\mathrm{~N} / \mathrm{m})$ & 0 & 0.018 & 0.069 & 0.159 & 0.287 \\
\hline$q_{4}(\mathrm{~N} / \mathrm{m})$ & 0 & 0.036 & 0.135 & 0.301 & 0.541 \\
\hline \multirow[t]{2}{*}{$N(\mathrm{~N})$} & $0.015^{\mathrm{a}}$ & $0.019^{\mathrm{a}}$ & $0.032^{\mathrm{a}}$ & $0.052^{\mathrm{a}}$ & $0.081^{\mathrm{a}}$ \\
\hline & 0.015 & 0.019 & 0.030 & 0.046 & 0.065 \\
\hline \multirow[t]{2}{*}{$\theta\left(^{\circ}\right)$} & $85.1^{\mathrm{a}}$ & $87.1^{\mathrm{a}}$ & $91.3^{\mathrm{a}}$ & $94.7^{\mathrm{a}}$ & $97.0^{\mathrm{a}}$ \\
\hline & 85.1 & 87.2 & 91.2 & 94.1 & 95.3 \\
\hline \multirow[t]{2}{*}{$\beta\left(^{\circ}\right)$} & $30.8^{\mathrm{a}}$ & $30.9^{\mathrm{a}}$ & $31.2^{\mathrm{a}}$ & $31.6^{\mathrm{a}}$ & $33.0^{\mathrm{a}}$ \\
\hline & 30.8 & 30.7 & 30.3 & 29.3 & 28.1 \\
\hline$T_{\mathrm{b}}^{*}(\mathrm{Nm})$ & 0.00165 & 0.00222 & 0.00374 & 0.00582 & 0.00823 \\
\hline \multirow{2}{*}{$T_{\mu}^{*}(\mathrm{Nm})$} & $0.00167^{\mathrm{a}}$ & $0.00215^{\mathrm{a}}$ & $0.00355^{\mathrm{a}}$ & $0.00578^{\mathrm{a}}$ & $0.00889^{\mathrm{a}}$ \\
\hline & 0.00167 & 0.00209 & 0.00328 & 0.00503 & 0.00715 \\
\hline \multirow[t]{2}{*}{$T_{\mathrm{ad}}^{*}(\mathrm{Nm})$} & $0^{\mathrm{a}}$ & $0.00007^{\mathrm{a}}$ & $0.00027^{\mathrm{a}}$ & $0.00059^{\mathrm{a}}$ & $0.00102^{\mathrm{a}}$ \\
\hline & 0 & 0.00026 & 0.00099 & 0.00227 & 0.00413 \\
\hline \multirow{2}{*}{$T_{\mu}^{*}+T_{\mathrm{ad}}^{*}(\mathrm{Nm})$} & $0.00167^{\mathrm{a}}$ & $0.00222^{a}$ & $0.00382^{\mathrm{a}}$ & $0.00637^{\mathrm{a}}$ & $0.00991^{\mathrm{a}}$ \\
\hline & 0.00167 & 0.00235 & 0.00427 & 0.00730 & 0.01128 \\
\hline \multirow{2}{*}{$T_{\mathrm{ad}}^{*} /\left(T_{\mu}^{*}+T_{\mathrm{ad}}^{*}\right)(\%)$} & $0^{\mathrm{a}}$ & $3.2^{\mathrm{a}}$ & $7.1^{\mathrm{a}}$ & $9.3^{\mathrm{a}}$ & $10.3^{\mathrm{a}}$ \\
\hline & 0 & 11.1 & 23.2 & 31.1 & 36.6 \\
\hline
\end{tabular}

Intensity $q\left(s^{\prime}\right)$, contact force $N$ and torque $T_{\mu}^{*}$ and $T_{\text {ad }}^{*}$ increase roughly quadratically as a function of the rotation speed. As to be expected, the torque $T_{\mathrm{ad}}^{*}$ (with $c=1$ ) is here lower than that given in Table 1. This is because of the more bended shape giving lower intensities and smaller moments. Air drag affects only slightly the deflection of the bristle at rotation speeds of 0-2000 rpm whereas the effect of air drag is rather significant on the torque $T^{*}$ (with $c=1$ ). It is to be noted that neither the normal force $N$ nor the centrifugal forces give any torque contributions as their lines of actions go through the origin. Without any discretization errors, we should have here in theory the result $T_{\mathrm{b}}^{*}=T_{\mu}^{*}+T_{\mathrm{ad}}^{*}$. Again, one should not rely much on the local values $T_{\mathrm{b}}^{*}$. 


\subsubsection{Effect of air drag on deflection of a rotating single bristle without contact}

Actually, not a single bristle but a bristle pair has been used in the experiments. This adds the possibility to take an average of the measurements. A small drawback is the increased air motion from the second bristle. A small rigid rod having the same direction as the bristle at the shaft is placed about $50 \mathrm{~mm}$ in front of the bristle to aid the measurements from the photographs. Figure 7 shows behaviour of the rotating bristle pair with the rotation speed of $1000 \mathrm{rpm}$ and $2000 \mathrm{rpm}$ in the clockwise direction.

(a)
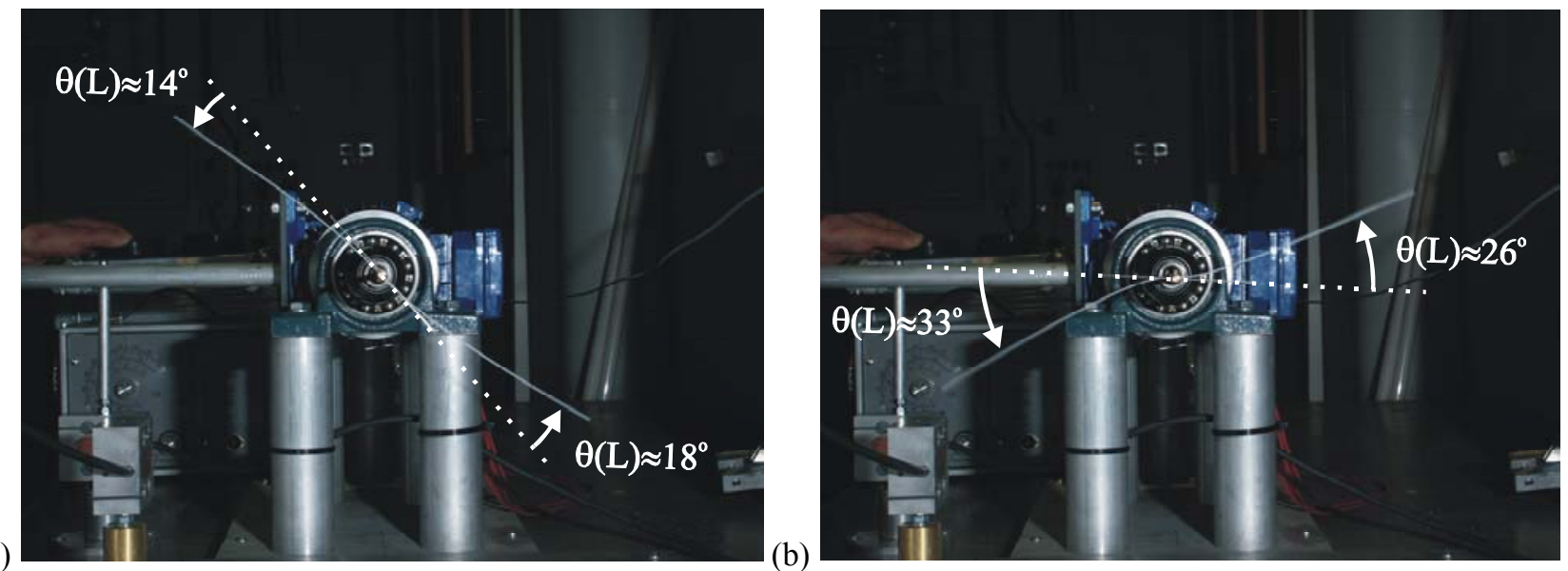

Fig. 7. Deflected form of the bristle (a) bristle rotation speed $n=1000 \mathrm{rpm}$ and (b) $n=2000 \mathrm{rpm}$ in air.

Comparison with the corresponding simulated results presented in Figure 5 shows rather satisfactory matching. Especially, the type of the deflected shape of the bristle obtained by simulation seems qualitatively correct.

Figures 8 (a) and 8 (b) present force $P$ from transducer 4 when the motor is rotated alone to obtain the bearing friction effect. Figures 8 (c) and 8 (d) show force $P$ due to bearing friction and air drag when the brush is rotated in air. 

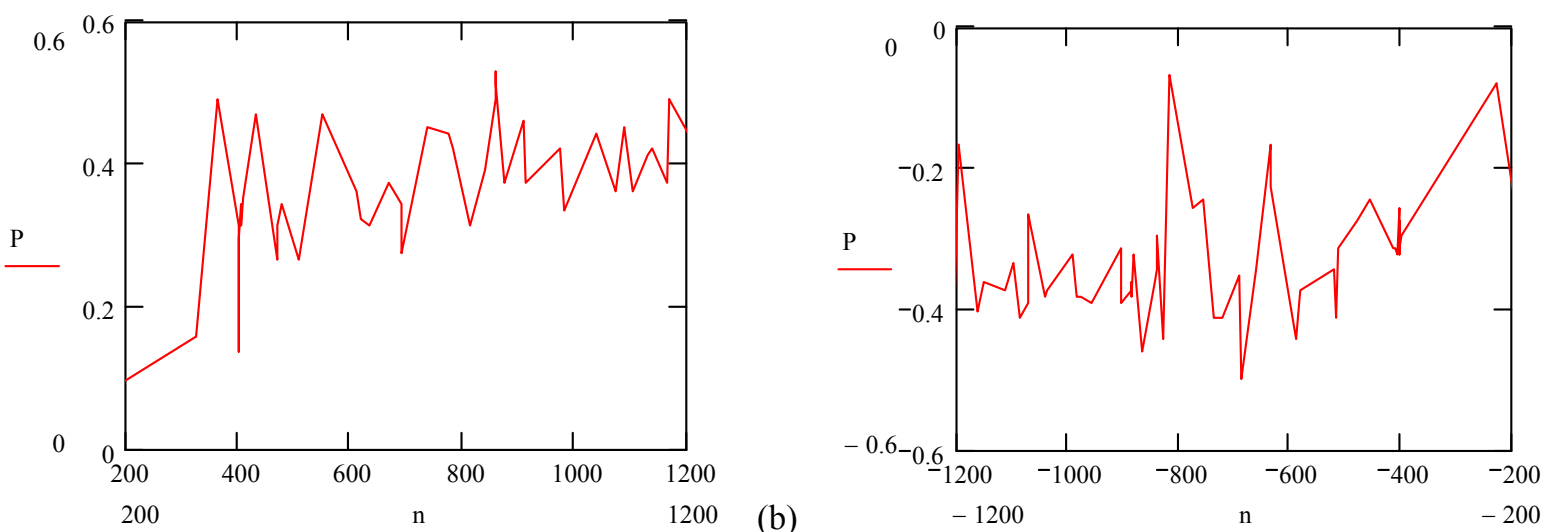

(a)

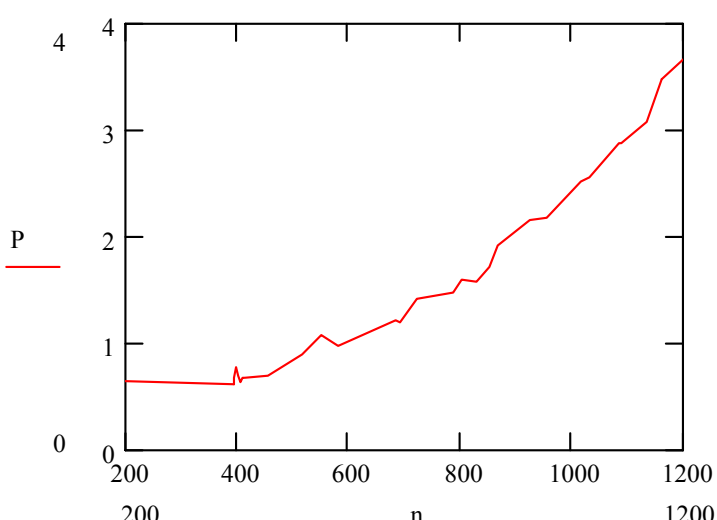

(c)

200

(d)

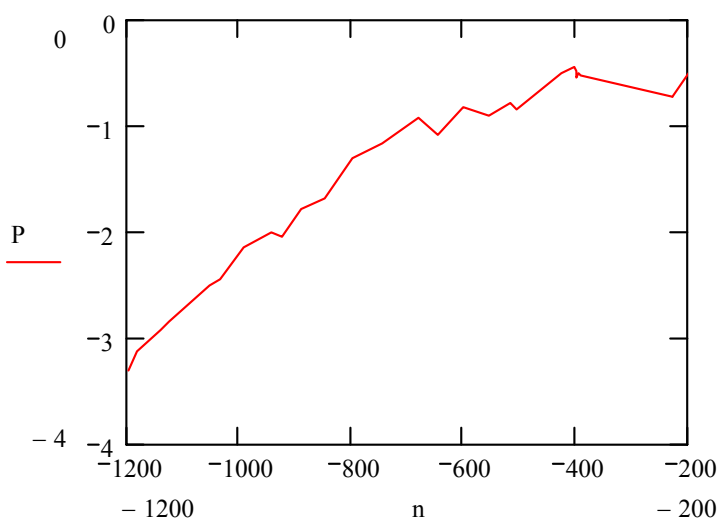

Fig. 8. (a) and (b) Force $P[\mathrm{~N}]$ without brush. (c) and (d) Force $P[\mathrm{~N}]$ when the brush rotates in air as functions of the rotation speed $n[\mathrm{rpm}]$.

Concerning the figures presenting experimental results in general we may note the following. The counterclockwise rotation direction is considered again positive with respect to the rotation speed $n$. No values are given when the magnitude of $n$ is under $200 \mathrm{rpm}$ as the measured data may be considered rather unreliable with the corresponding small forces. The oscillations in the data are mainly due to the unavoidable vibration generated into the test system. For most quantities presented here the magnitudes should be in theory equal for a given magnitude of $n$ in the counterclockwise and clockwise directions. In practise, especially the brush has some directional bias due to its construction (see Figure 10 (b)). Further, the bristle tips wear in contact and may also show directional asymmetry. 
Figures 9 (a) and 9 (b) present the magnitude of torque $T_{\text {ad }}$ due to air drag evaluated from measuring results (equation (24)) when the brush is rotated in air as functions of the rotation speed. Additionally, the torque from the simulation using the values $c=0.5$ and $c=1$ calculated from (see equation (32))

$T_{\mathrm{ad}}=n T_{\mathrm{ad}}^{*}$

is shown in Figures 9 (a) and 9 (b).

(a)
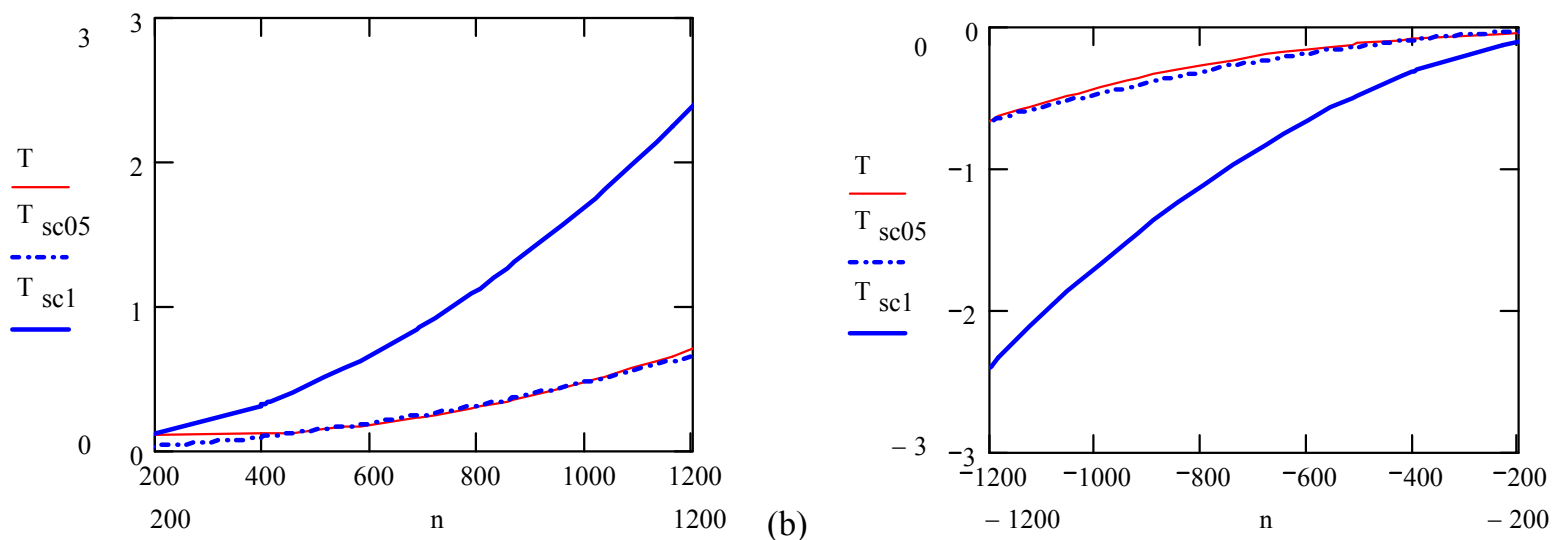

Fig. 9. The measured $T[\mathrm{Nm}]$ and simulated torque $T_{\mathrm{s}}[\mathrm{Nm}]$ due to air drag with $c=0.5$ and $c=1$ when the brush rotates in air as functions of the rotation speed $n$ [rpm].

The results of the laboratory test and the simulation are nearly identical with $c=0.5$ in the simulation. With $c=1$, the simulation gives too large values which was to be expected due to the bulk air motion found to be present in the experiments.

\subsubsection{Effect of air drag on deflection of rotating bristle and brush in duct}

Figure 10 shows deflection of the bristle pair and the brush with the rotation speeds of $0 \mathrm{rpm}$, $500 \mathrm{rpm}$ and $1000 \mathrm{rpm}$ (clockwise direction) in the duct. 
(a)

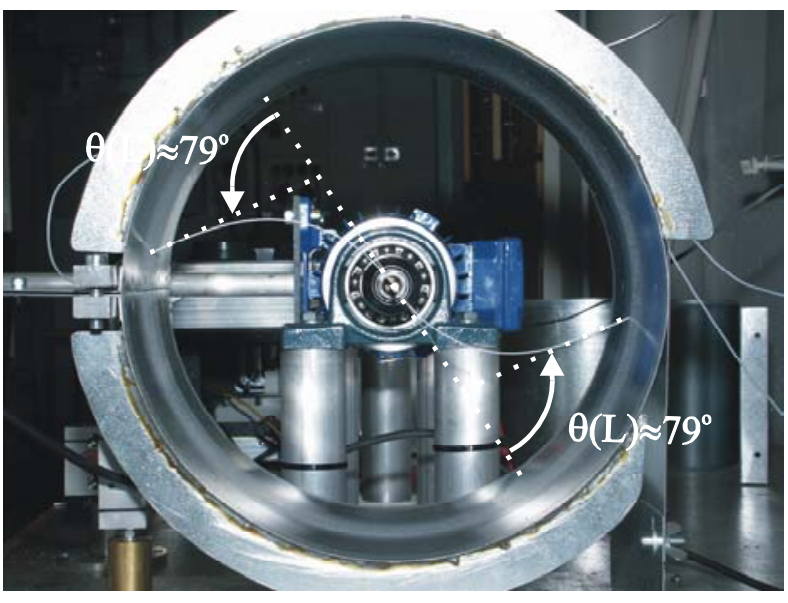

(b)

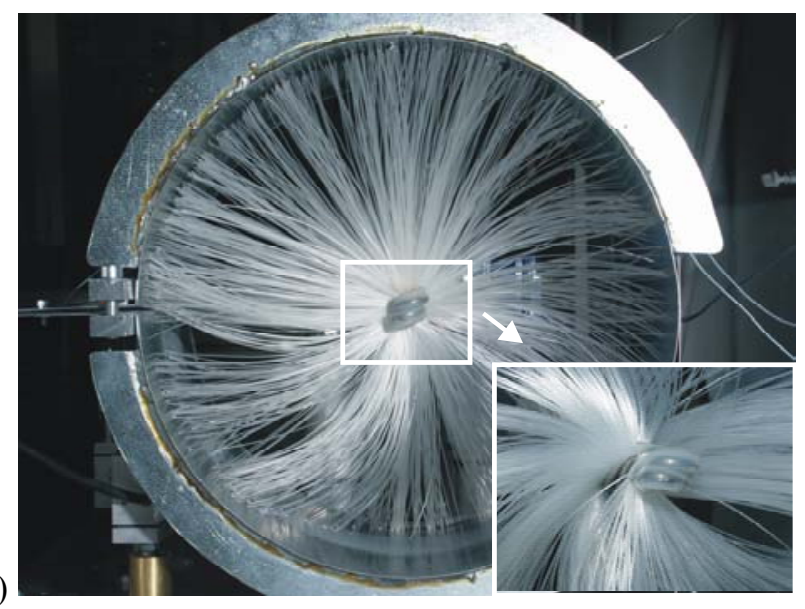

(c)

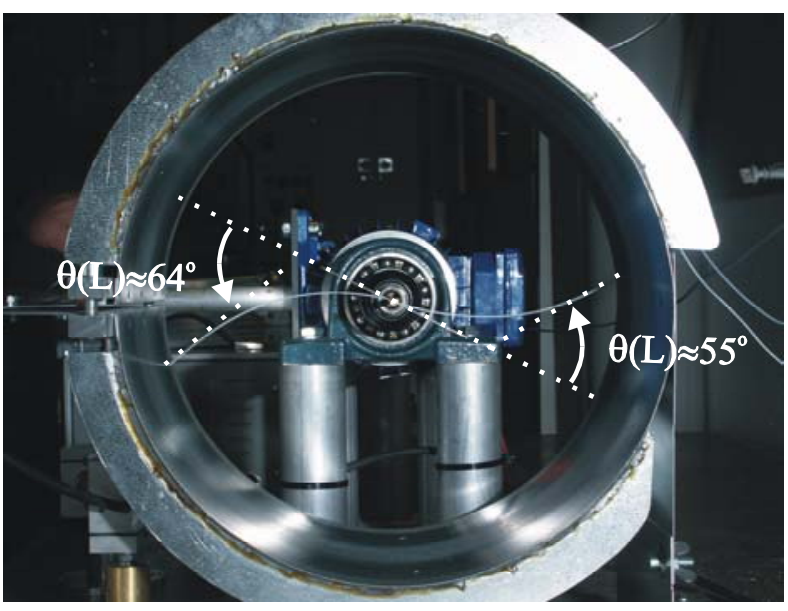

(d)
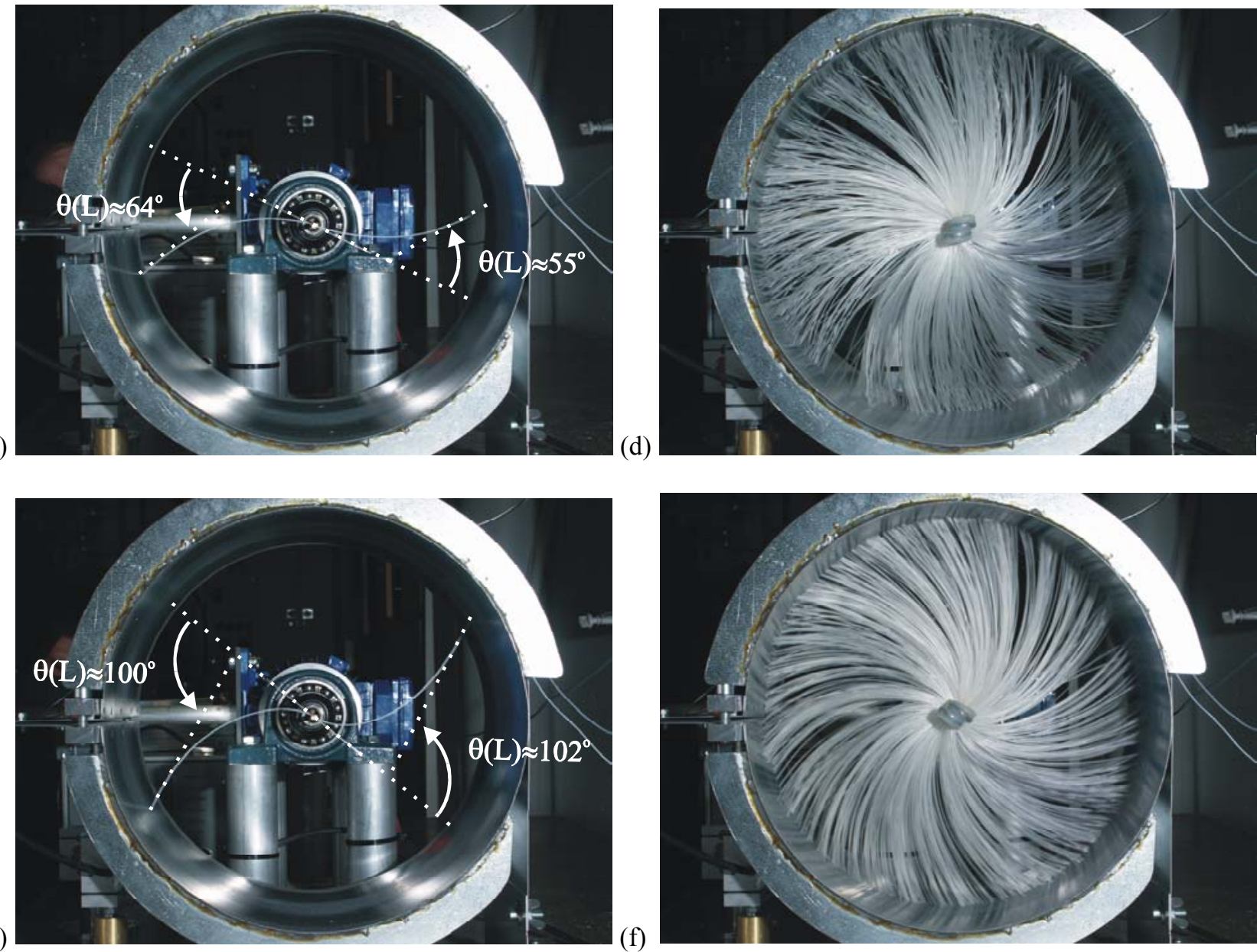

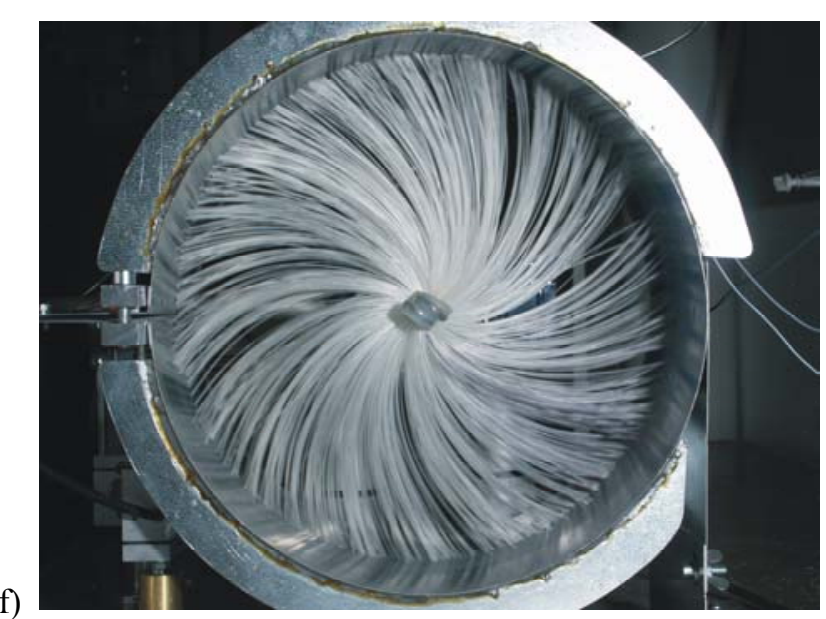

Fig. 10. Deflected form of the bristle (a) bristle rotation speed $n=0 \mathrm{rpm}$, (c) $n=500 \mathrm{rpm}$ and (e) $n=1000 \mathrm{rpm}$ in the duct with contact. Deflected form of the brush (b) brush rotation speed $n=0 \mathrm{rpm}$, (d) $n=500 \mathrm{rpm}$ and (f) $n=1000 \mathrm{rpm}$ in the duct with contact.

The deflection of the bristle pair is similar same to that obtained by the simulation (Table 2) when $n=0 \mathrm{rpm}$ and $n=1000 \mathrm{rpm}$. At rotation speed $n=500 \mathrm{rpm}$ the bristle pair was found not to remain in the plane assumed in the simulation and therefore the deflection of the bristle pair seen in 
the photograph is low compared to that obtained by the simulation. The deflections of bristles of the brush are difficult to estimate accurately because the bristles were connected asymmetrically on a spiral frame made of metal wire (Figure $10(\mathrm{~b})$ ).

Figures 11 (a) and 11 (b) give the measurement results for force $P$ (transducer 4), Figures 11 (c) and 11 (d) for force $B$ (transducer 1) and Figures 11 (e) and 11 (f) for force $E$ (transducers 2 and 3) as functions of the rotation speed, when the brush is in contact with the duct. 

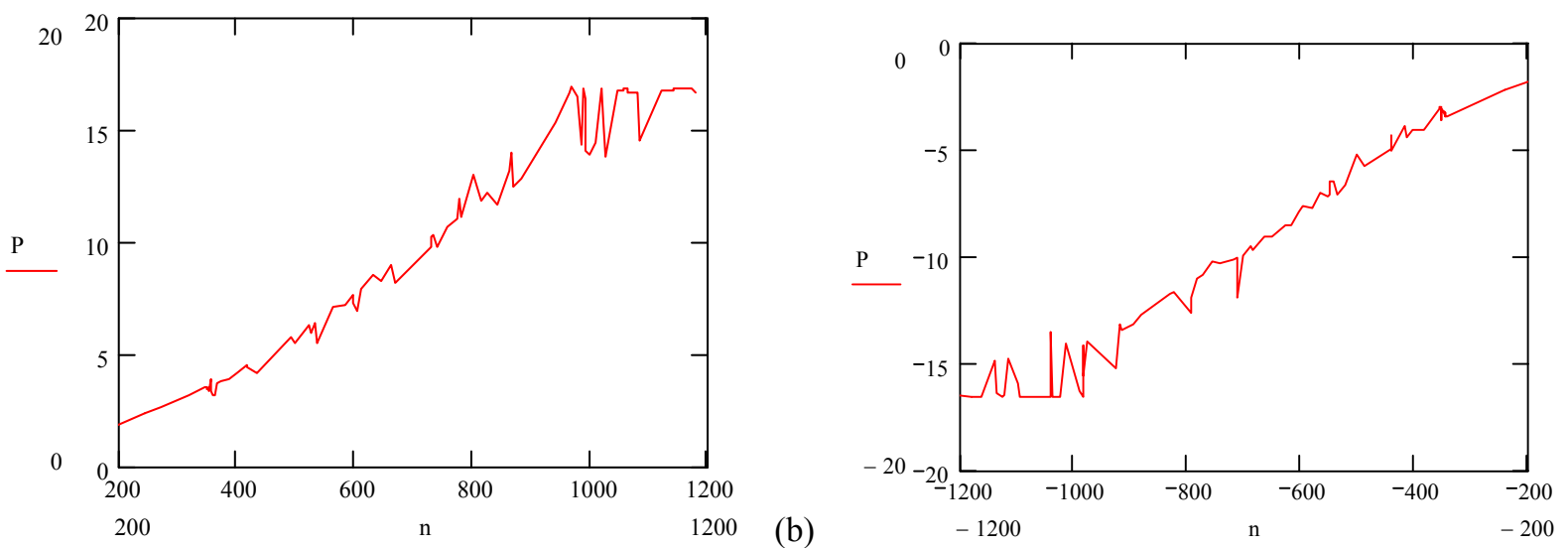

(a)

(b)
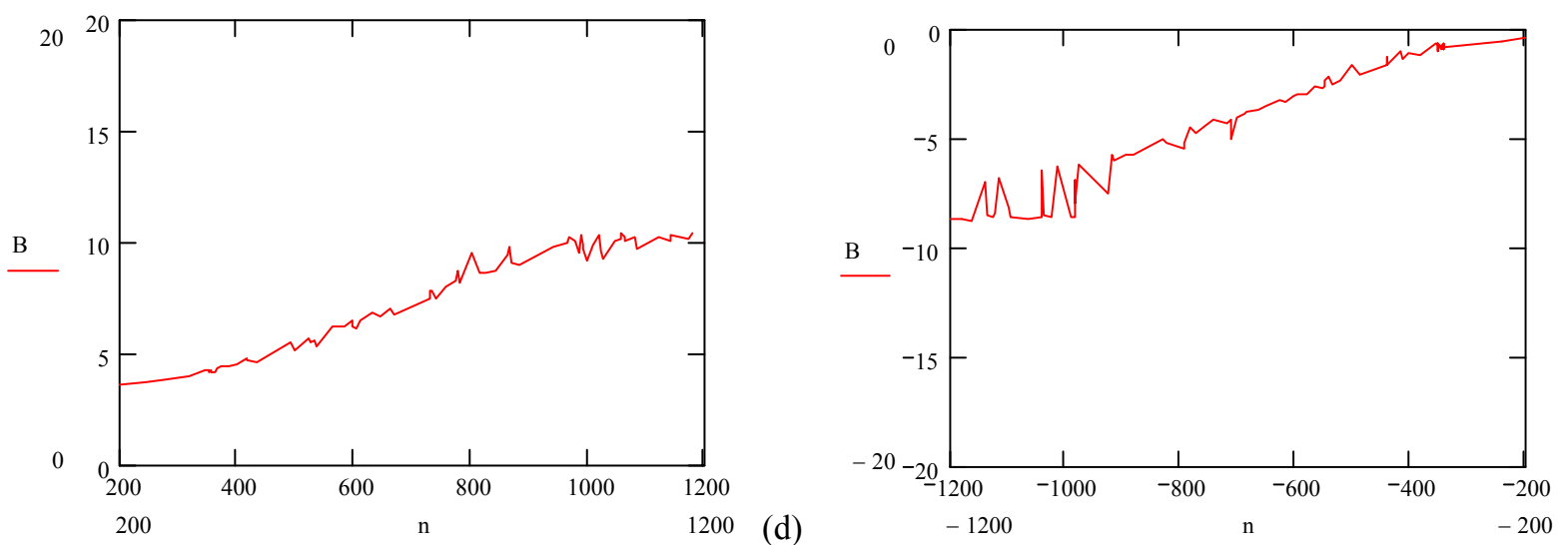

(c)

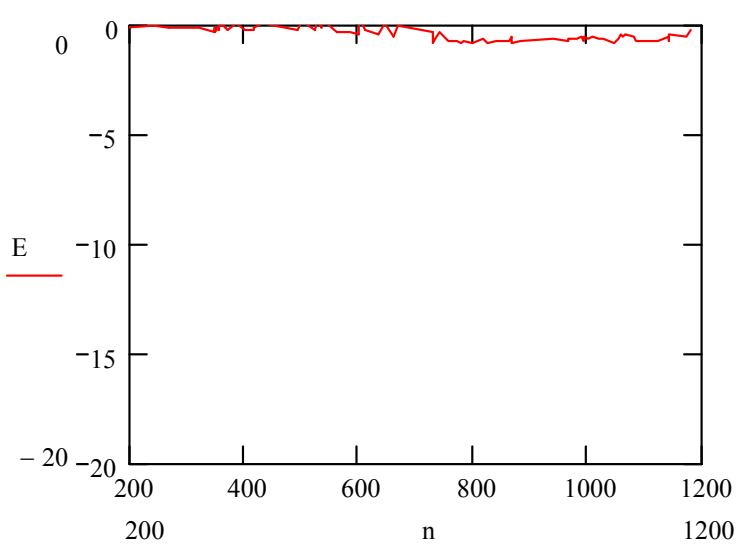

(d)

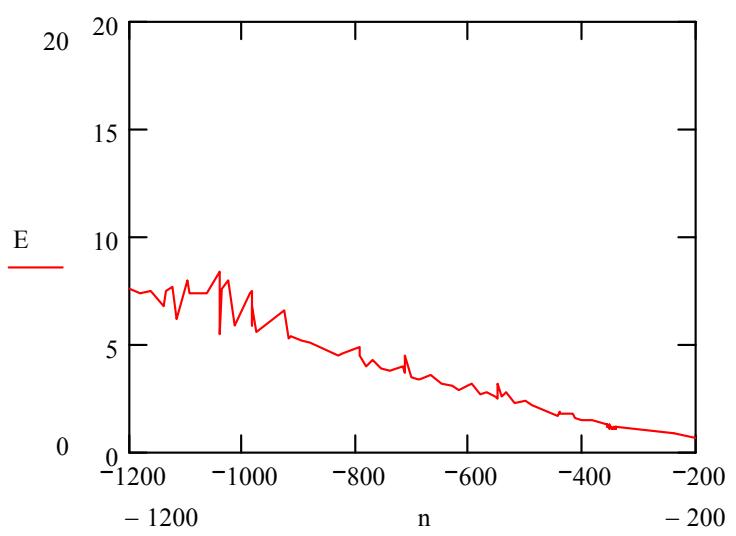

Fig. 11. The magnitude of forces $P[\mathrm{~N}], B[\mathrm{~N}]$ and $E[\mathrm{~N}]$ as functions of the rotation speed $n[\mathrm{rpm}]$.

The behaviour of force $E$ with respect to rotation direction is qualitatively in accordance with the theory. From equation (26), friction coefficient has to be higher than 0.64 if $E$ becomes negative when the brush is rotated in the counterclockwise direction (Figure $11(\mathrm{e})$ ). For low magnitudes of $n$, there is quite a large difference in the magnitudes of force $B$ with respect to the rotation direction; in theory the magnitudes should be equal. 
Figure 12 presents the brush tip normal force $N$ evaluated from measured data by Equations (25) and (28) as well as the simulated results $N_{\mathrm{s}}$ with $c=0.5$ and $c=1$.

(a)
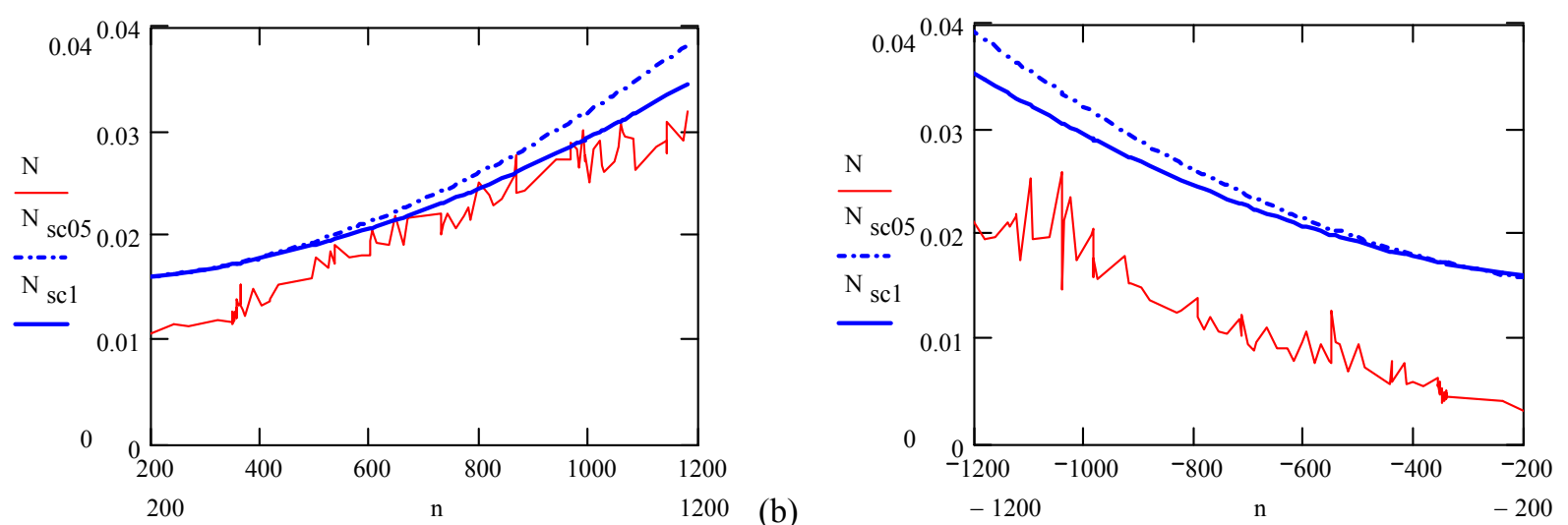

Fig. 12. The measured $N[\mathrm{~N}]$ and simulated normal force $N_{\mathrm{s}}[\mathrm{N}]$ with $c=0.5$ and $c=1$ as functions of the rotation speed $n[\mathrm{rpm}]$.

The magnitudes of the experimental and simulated values are roughly of the same order. However, there is quite a difference in the experimental results with respect to rotation direction and it is difficult to judge which results are more reliable. The possible deviations of the bristles from the plane (see Figure 2) assumed in the simulation may explain the somewhat lower values obtained in the experiment.

Figures 13 (a) and 13 (b) present torque $T_{\text {ad }}$ due to air drag evaluated from the measuring results (equation (23)) and the results of the simulation (equation (34)) with $c=0.5$ and $c=1$ as a function of rotation speed. 


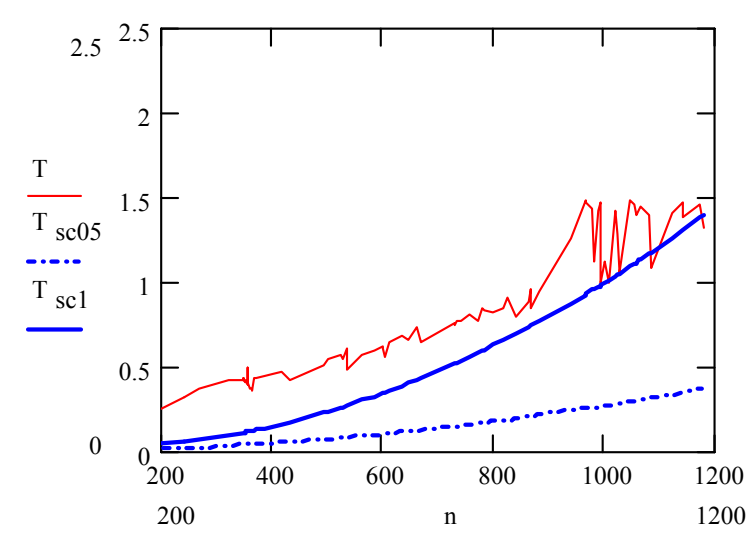

(a)

Fig. 13. The measured torque $T[\mathrm{Nm}]$ and simulated torque $T$ functions of the rotation speed $n$ [rpm].

The torque from evaluated measured data $(P$ and $B)$ gives values which must be considered too high as they are higher than those recorded in Figure 9 for the brush in air. It may be noted that when the brush was rotated in air without contact in a duct with a diameter of $0.365 \mathrm{~m}$ the torque due to air drag was lower than without the duct. This further seems to indicate that the values obtained are too high. One explanation for these inconsistent results may be in form of formula (23). Subtraction of oscillating experimental values of the same order of magnitude from each other can obviously lead to rather large errors. It is also seen that the results by the measurements deviate quite a lot with respect to rotation direction. The simulated results with $c=1$ seem to tend to overestimate the air drag with higher rotation speeds. The "slopes" of measured and simulated curves with $c=0.5$ seem to be rather close to each other.

Figure 14 presents friction coefficient $\mu$ evaluated by Equations (26) and (29) as a function of rotation speed. 

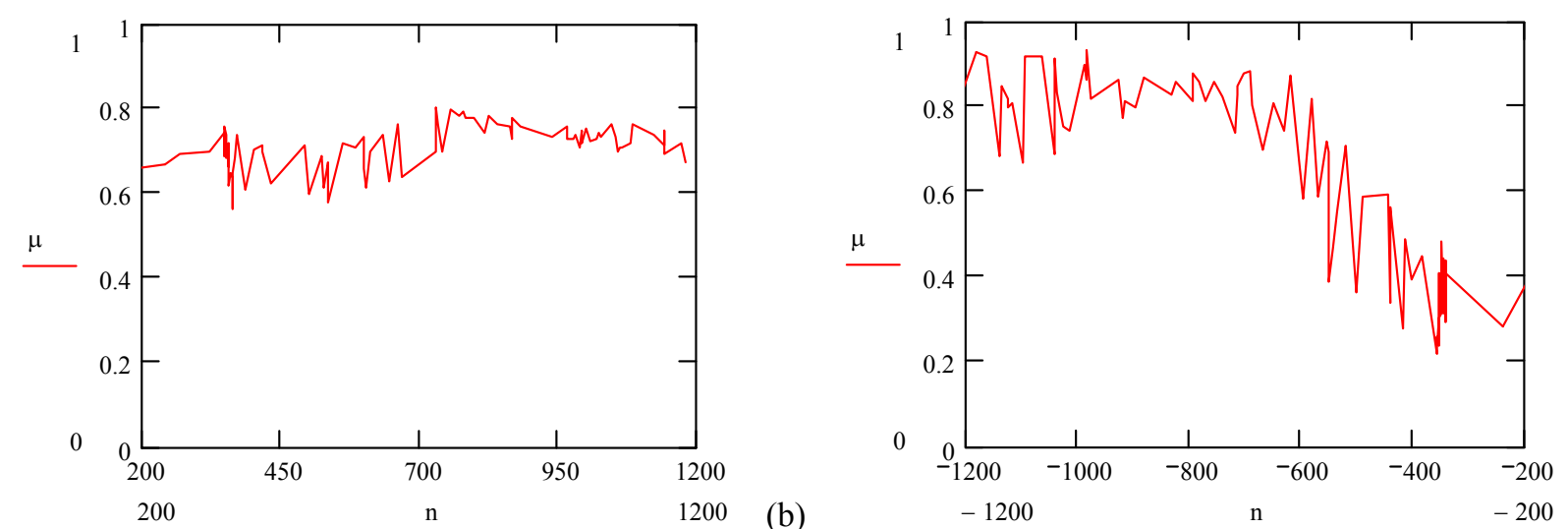

(a)

Fig. 14. The friction coefficient $\mu[-]$ as functions of the rotation speed $n[\mathrm{rpm}]$.

The reason for low values for $\mu$ obtained for low rotation speeds in the clockwise direction may be based on the inconsistent behaviour of force $B$ discussed in connection with Figure 11. In any case, the average values (roughly 0.7 and 0.65 ) are clearly higher than those (roughly 0.5 ) obtained for nylon in [12] with a different test arrangement. Because of the high friction coefficient the tips of the bristle pair were worn rapidly when the rotation speed was higher than $2000 \mathrm{rpm}$. Based on the results in Figure 14, we have used the value $\mu=0.7$ in the simulations.

\section{Conclusion}

The experimental results obtained in general give some confidence on the validity of the proposed simulation model so that it can be used in analysing and in comparing different brush designs. In article [4], by the present authors, a coding error was later found concerning the air drag integrals. The present article gives corrected results in this respect. However, the main conclusion presented earlier remain is valid. As expected, the model overestimates the effect of air drag of a brush with $c=1$. These results are thus "on the safe side" with respect to the power need. A value $c=0.5$ was found to give a good agreement with experimental results in one test case. However, this value will not necessarily work well with other brush types. The torque increases roughly quadratically at the rotation speed of 300-1000 rpm due to air drag. Because of the air drag both the magnitude of the normal force and the contact angle decrease when the rotating speed of the bristle increases. 
Therefore, it can be recommended that the rotating speed of the brush should be adjustable. I conclusion, the Mathcad software seems to be a useful tool in the cleaning brush design work and to solve the highly nonlinear problem connected to the model.

\section{Acknowledgements}

This study was carried out as a part of the Lifa Duct Cleaning Concept funded by the Lifa Air Ltd and the National Technology Agency of Finland (Tekes). The authors would like to thank especially laboratory manager Iikka Järvenpää for his valuable comments for the laboratory test and laboratory manager Markku Sivukari for arrangements for the laboratory test. 


\section{Nomenclature}

$\begin{array}{ll}A & \text { cross-sectional area of bristle } \\ c & \text { dimensionless multiplier } \\ c_{0}, c_{1}, \ldots, c_{n} & \text { undetermined parameters } \\ C_{\mathrm{D}} & \text { air drag coefficient } \\ d & \text { thickness of bristle } \\ \mathrm{d} m & \text { differential mass element of bristle } \\ \mathrm{d} m \omega^{2} x^{\prime} & \text { differential centrifugal force component acting on mass element in } x \text {-axis } \\ \mathrm{d} m \omega^{2} y^{\prime} & \text { direction } \\ \mathrm{d} s & \text { differential centrifugal force component acting on mass element in } y \text {-axis } \\ E & \text { direction } \\ E I & \text { differential bristle length element } \\ L & \text { Young's modulus of bristle material } \\ M & \text { flexural rigidity of bristle } \\ n & \text { length of bristle } \\ N & \text { bending moment } \\ P & \text { rotation speed of bristle, number of bristles in brush } \\ q & \text { magnitude of normal force } \\ Q & \text { cross-sectional normal force, force measured by transducer } 4 \\ R & \text { air drag force per unit bristles length acting perpendicular to bristle } \\ S & \text { shearing force } \\ S_{1}, s_{2}, \ldots, s_{m} & \text { radius of duct } \\ T & \text { arc length coordinate along bristle } \\ v & \text { interpolation points in interval } 0 \leq s \leq L \\ x, y & \text { torque } \\ x^{\prime}, y^{\prime} & \text { speed of cylinder with respect to air } \\ & \text { coordinates of a generic point of bristle } \\ & \text { coordinates of a generic point inside end part of bristle }\end{array}$

Greek symbols

$\alpha$

$\beta$

$\gamma$

$\theta$

$\widetilde{\theta}$

$\mu$

$v_{\mathrm{a}}$

$\rho_{\mathrm{a}}$

$\rho_{\mathrm{b}}$

$\phi$

$\omega$ polar angle

bristle tip contact angle

angle between rod normal and rod velocity vector

inclination angle of bristle axis with $x$-axis

approximation of $\theta$

kinetic coefficient of friction

kinematic viscosity of air

density of air

density of bristle material

inclination angle of a ray to $s$ to with respect to $x$-axis

angular speed of brush 


\section{References}

[1] Holopainen R, Pasanen P, Seppänen O. Duct Cleanliness in New HVAC Installations. Proceedings of Healthy Buildings 2000 Conference. The $6^{\text {th }}$ Healthy Buildings Conference. Vol. 2, pp. 175-180. Espoo, Finland.

[2] Pasanen P. Emissions from the Filters and Hygiene of Air Ducts in the Ventilation Systems of Office Buildings. Department of Environmental Sciences. Doctoral dissertation. University of Kuopio. 1998.

[3] Holopainen R, Asikainen V, Tuomainen M, Björkroth M, Pasanen P, Seppänen O. Effectiveness of duct cleaning methods on newly installed duct surfaces. Indoor Air 13 (2003) $212-222$.

[4] Holopainen R, Salonen E-M. Modelling the cleaning performance of rotating brush in duct cleaning. Helsinki University of Technology. Energy and Buildings 8 (2002) 845-852.

[5] Holopainen R, Salonen E-M. Modelling bristle behaviour in rotating brush duct cleaning. Helsinki University of Technology. Department of Mechanical Engineering. Laboratory of Heating, Ventilating and Air Conditioning. Report B75. 58 p. 2003.

[6] Timoshenko SP, Gere JM. Theory of Elastic Stability. McGraw-Hill Book Company 1961.

[7] Schlichting H. Boundary-Layer Theory. McGraw-Hill Book Company 1979.

[8] Blevins R. Flow-Induced Vibration. Van Nostrand Reinhold International Company Limited 1990.

[9] http://www.mathcad.com/ (4 ${ }^{\text {th }}$ April, 2003).

[10] Martonen P. Puhdistusharjan ilmanvaihtokanavaan synnyttämien voimien kokeellinen määritys. (Experimental determination of the forces from a rotating brush in a ventilation duct). Individual Assignments in Strength of Materials. Laboratory for Mechanics of Materials. Helsinki University of Technology. 2002. In Finnish. 
[11] Viljamaa A, Eriksson L. Polyamidin E-modulin mittaaminen. (Polyamide Young's modulus and its measuring in the laboratory). The Laboratory of Processing and Heat Treatment of Materials. Helsinki University of Technology. 2003. In Finnish. Private communication.

[12] Rautiainen S. Harjaksen ja teräspellin välinen kitka. (Friction coefficient between bristle and steel plate). Department of Applied Physics. University of Kuopio. 2002. In Finnish. 